\title{
Optimizing Range Query Processing for Dual Bitmap Index ${ }^{\dagger}$
}

\author{
Naphat KEAWPIBAL, Ladda PREECHAVEERAKUL and \\ Sirirut VANICHAYOBON*
}

Department of Computer Science, Faculty of Science, Prince of Songkla University, Songkhla 90110, Thailand

('Corresponding author’s e-mail: sirirut.v@psu.ac.th)

Received: 16 June 2017, Revised: 10 December 2017, Accepted: 19 December 2017

\begin{abstract}
Bitmap-based indexes are known to be the most effective indexing method for retrieving and answering selective queries in a read-only environment. Various types of encoding bitmap indexes significantly improve query time efficiency by utilizing fast Boolean operations directly on the index before retrieving the raw data. In particular, the dual bitmap index improves the performance of equality queries in terms of the space vs. time trade-off. However, the performance of range queries is unsatisfactory. In this paper, an optimizing algorithm is proposed to improve the range query processing for the dual bitmap index. The results of the experiment conducted show that the proposed algorithm, called Dual-simRQ, reduces the number of bitmap vectors scanned and the Boolean operations performed, which impacts the overall performance for range query processing.
\end{abstract}

Keywords: Encoding bitmap indexes, dual bitmap index, range query processing, Dual-simRQ

\section{Introduction}

Advances in technology have caused a data explosion and a massive amount of data is generated by various devices [1-3]. The problem of data management, including storage requirements and query processing, has become more difficult. The issue of query performance has been extensively studied, especially in regard to data warehouses and scientific data management applications [3-6]. Generally, a data warehouse is deployed as a read-only environment and is used to support forecasting and decisionmaking applications [3-5,7,8]. Therefore, a lot of complex queries (i.e., equality, range, and membership queries) are submitted to data warehouses in order to retrieve data and operate further processes. Due to the massive amount of data and the complexity of some queries, a long time might be taken to process a query before a result can be returned [4,5]. As a result, query performance and reducing query processing time have become an important issue in data warehousing.

Indexing is the best-known method of speeding up search operations and the retrieval process, without requiring additional hardware [4,9]. Indexing methods can be categorized as hash-based indexes, tree-based indexes, and bitmap-based indexes [10-13]. In particular, it is easy to represent data in a binary format in bitmap-based indexes and query processing can be executed extremely fast by applying the Boolean operators, AND, OR, NOT, and XOR, directly on the index before accessing raw data [4,5]. A simple bitmap index [14] was first introduced and recommended for space- and time-efficiency for the attribute with low cardinality, for example, the attribute, sex which has a cardinality of 2. Unfortunately, when the cardinality is high, the size of a bitmap index is significantly increased which leads to a problem

\footnotetext{
${ }^{\dagger}$ Presented at the $14^{\text {th }}$ International Joint Conference on Computer Science and Software Engineering: July $12^{\text {th }}-14^{\text {th }}$, 2017
} 
http://wjst.wu.ac.th

with the bitmap index space requirement. To deal with this problem, several strategies have been employed to improve the simple bitmap index which can be divided into 4 categories [6,9,10,15-17]: binning, compressing, encoding, and multi-level and multi-component. Among these 4, encoding strategies relate to encoding schemes for representing data by using a small number of bitmap vectors while maintaining the query processing time.

A number of sophisticated encoding bitmap indexes have been proposed in the last 10 years, including the range bitmap index [15,18], interval bitmap index [15], encoded bitmap index [19], scatter bitmap index [20,21], and dual bitmap index [22]. Among these 5 encoding bitmap indexes, the range bitmap index and interval bitmap index produce a large number of bitmap vectors when dealing with high cardinality attributes, which is likely to have an impact on the space requirement, while the encoded bitmap index generates the least number of bitmap vectors but its query processing time is unsatisfactory [23-26]. On the other hand, the dual bitmap index requires a smaller number of bitmap vectors than the range, interval, and scatter bitmap indexes. Specifically, to answer an equality query in the form " $A=v$ ", the dual bitmap index uses 2 bitmap vectors and one bitwise-AND operation. As a result, the query processing time of the dual bitmap index is extremely fast. Further, to answer a range query in the form " $v_{1} \leq A \leq v_{2}$ ", each of 2 bitmap vectors is accessed and performed by a bitwise-AND operation to obtain each query value, and then, bitwise-OR operations are used to get the final result of the query. The dual bitmap index has therefore been demonstrated to improve query performance for an equality query from the point of view of a trade-off between space required and time consumed [22]. In contrast, the query performance for a range query is unsatisfactory.

In this paper, an algorithm is presented to improve the query performance of range query processing for the dual bitmap index, namely the Dual-simRQ. The objective of the algorithm is to simplify the retrieval function by eliminating unnecessary scanning of bitmap vectors and bitwise operations. The algorithm provides less complexity of the retrieval function which is an impact on the execution time required for accessing bitmap vectors as well as the time used for bitwise operations.

\begin{tabular}{cc}
\hline RID & $A$ \\
\hline 1 & 3 \\
2 & 9 \\
3 & 14 \\
4 & 8 \\
5 & 10 \\
6 & 3 \\
7 & 4 \\
8 & 0 \\
9 & 12 \\
10 & 5 \\
11 & 13 \\
12 & 6 \\
13 & 0 \\
$\cdot$ & $\cdot$ \\
$\cdot$ & $\cdot$ \\
$\cdot$ & $\cdot$ \\
100,000 & 2 \\
\hline
\end{tabular}

(a)

\begin{tabular}{ccccccccccccccc}
\hline$S^{0}$ & $S^{1}$ & $S^{2}$ & $S^{3}$ & $S^{4}$ & $S^{5}$ & $S^{6}$ & $S^{7}$ & $S^{8}$ & $S^{9}$ & $S^{10}$ & $S^{11}$ & $S^{12}$ & $S^{13}$ & $S^{14}$ \\
\hline 0 & 0 & 0 & 1 & 0 & 0 & 0 & 0 & 0 & 0 & 0 & 0 & 0 & 0 & 0 \\
0 & 0 & 0 & 0 & 0 & 0 & 0 & 0 & 0 & 1 & 0 & 0 & 0 & 0 & 0 \\
0 & 0 & 0 & 0 & 0 & 0 & 0 & 0 & 0 & 0 & 0 & 0 & 0 & 0 & 1 \\
0 & 0 & 0 & 0 & 0 & 0 & 0 & 0 & 1 & 0 & 0 & 0 & 0 & 0 & 0 \\
0 & 0 & 0 & 0 & 0 & 0 & 0 & 0 & 0 & 0 & 1 & 0 & 0 & 0 & 0 \\
0 & 0 & 0 & 1 & 0 & 0 & 0 & 0 & 0 & 0 & 0 & 0 & 0 & 0 & 0 \\
0 & 0 & 0 & 0 & 1 & 0 & 0 & 0 & 0 & 0 & 0 & 0 & 0 & 0 & 0 \\
1 & 0 & 0 & 0 & 0 & 0 & 0 & 0 & 0 & 0 & 0 & 0 & 0 & 0 & 0 \\
0 & 0 & 0 & 0 & 0 & 0 & 0 & 0 & 0 & 0 & 0 & 0 & 1 & 0 & 0 \\
0 & 0 & 0 & 0 & 0 & 1 & 0 & 0 & 0 & 0 & 0 & 0 & 0 & 0 & 0 \\
0 & 0 & 0 & 0 & 0 & 0 & 0 & 0 & 0 & 0 & 0 & 0 & 0 & 1 & 0 \\
0 & 0 & 0 & 0 & 0 & 0 & 1 & 0 & 0 & 0 & 0 & 0 & 0 & 0 & 0 \\
1 & 0 & 0 & 0 & 0 & 0 & 0 & 0 & 0 & 0 & 0 & 0 & 0 & 0 & 0 \\
. &. &. &. &. &. &. &. &. &. &. &. &. &. &. \\
. &. &. &. &. &. &. &. &. &. &. &. &. &. &. \\
. &. &. &. &. &. &. &. &. &. &. &. &. &. &. \\
0 & 0 & 1 & 0 & 0 & 0 & 0 & 0 & 0 & 0 & 0 & 0 & 0 & 0 & 0
\end{tabular}

(b)

\begin{tabular}{cccccc}
\hline$D^{0}$ & $D^{1}$ & $D^{2}$ & $D^{3}$ & $D^{4}$ & $D^{5}$ \\
\hline 0 & 1 & 0 & 0 & 0 & 1 \\
0 & 0 & 1 & 1 & 0 & 0 \\
1 & 1 & 0 & 0 & 0 & 0 \\
1 & 0 & 0 & 0 & 1 & 0 \\
0 & 1 & 0 & 1 & 0 & 0 \\
0 & 1 & 0 & 0 & 0 & 1 \\
1 & 0 & 0 & 0 & 0 & 1 \\
0 & 0 & 0 & 0 & 1 & 1 \\
0 & 1 & 1 & 0 & 0 & 0 \\
0 & 0 & 0 & 1 & 1 & 0 \\
1 & 0 & 1 & 0 & 0 & 0 \\
0 & 0 & 1 & 0 & 1 & 0 \\
0 & 0 & 0 & 0 & 1 & 1 \\
$\cdot$ &. &. &. &. &. \\
. &. &. &. &. &. \\
. &. &. &. &. &. \\
0 & 0 & 1 & 0 & 0 & 1 \\
\hline
\end{tabular}

(c)

Figure 1 An example of Table $T$ (a) a simple bitmap index (b) and a dual bitmap index on attribute $A$ with $C=15$ (c).

\section{Literature review}

\section{Simple bitmap index [14]}

As mentioned above, a simple bitmap index is a well-known method of indexing which improves the speed of query processing with stored data, especially in a data warehouse. The simple bitmap index 
http://wjst.wu.ac.th

consists of $C$ bitmap vectors, where $C$ is the number of distinct values in the indexed attribute (cardinality), with each bitmap vector corresponding to one precise value of the indexed attribute. To represent value ' $v$ ', the $\mathrm{i}^{\text {th }}$ bit in the bitmap vector representing value ' $v$ ' is set to 1 if the $\mathrm{i}^{\text {th }}$ row of the indexed attribute contains value ' $v^{\prime}$, otherwise, the bit is set to 0 .

Assume that domain of attribute $A$ given by table $T$ is $\{0,1,2, \ldots, 14\}$, as shown in Figure 1(a). The simple bitmap index consists of 15 bitmap vectors. As shown in Figure 1(b), for example, in bitmap vector $S^{3}$ used for representing value ' $3^{\prime}$, the $1^{\text {st }}$ and $6^{\text {th }}$ bit in $S^{3}$ are set to 1 . When answering equality queries, one bitmap vector associated with the query value is scanned without performing any bitwise operations. For example, in order to evaluate the equality query " $A=3$ ", the bitmap vector $S^{3}$ is scanned. The row that contains bit 1 is returned as the result of this query, i.e., the $1^{\text {st }}$ and $6^{\text {th }}$ rows. However, when range queries are submitted, there are more than one bitmap vectors read, associated with the query values, and a bitwise-OR operation is performed on them before answering the query. For example, in order to evaluate the range query " $3 \leq A \leq 13$ ", the bitmap vectors between $S^{3}$ to $S^{13}$ are scanned. Then, bitwise-OR operations are performed among those bitmap vectors as $S^{3} \vee S^{4} \vee S^{5} \vee S^{6} \vee S^{7} \vee S^{8} \vee S^{9} \vee$ $S^{10} \vee S^{11} \vee S^{12} \vee S^{13}$ to obtain the final result. Table $T$ in Figure 1(a) is used as the example for the remainder of this paper.

\section{Dual bitmap index [22]}

The key design of a dual bitmap index is the use of 2 bitmap vectors to represent indexed attribute values by computing the values of $r$ and $s$ using Eqs. (1) and (2), respectively. The dual bitmap index consists of $[\sqrt{2 C+0.25}+0.5\rceil$ bitmap vectors.

$r=\lceil\sqrt{2(H i C-v)+0.25}+0.5\rceil$

$s=\left\lceil r-1-\left[\left(\frac{(n-r)(n-r-1)}{2}\right) \bmod r\right]\right\rceil$

where $v$ is the attribute value of any row, $n$ is the total number of bitmap vectors created, and $\operatorname{HiC}=\left(\begin{array}{l}n \\ 2\end{array}\right)$

By using Eq. (3) to represent attribute values, each value is identified by 2 bitmap vectors, i.e., $D^{r}$ and $D^{s}$. If the value at $\mathrm{i}^{\text {th }}$ row corresponds to the values of $r$ and $s$, the bit at $\mathrm{i}^{\text {th }}$ row of $D^{r}$ and $D^{s}$ is set to 1. Otherwise, it is set to 0 .

$D^{j}=\left\{\begin{array}{l}1, j=r \text { and } j=s, \\ 0, \text { otherwise. }\end{array}\right.$

For example, using table $T$, the dual bitmap index consists of 6 bitmap vectors, $\left\{D^{0}, D^{1}, D^{2}, D^{3}, D^{4}, D^{5}\right\}$. To represent value ' $3^{\prime}$ at the first row of attribute $A$, the $1^{\text {st }}$ bit of $D^{5}$ and $D^{1}$ is set to 1 since the values of $r$ and $s$ are 5 and 1 , respectively, while every $1^{\text {st }}$ bit remaining in the other bitmap vectors is set to 0 . Figure 1(c) shows a complete dual bitmap index created on attribute $A$ with 15 cardinalities. The evaluation of an equality query in the dual bitmap index performs the retrieval function as in Eq. (4).

$" A=v^{\prime}=D^{r} \wedge D^{s}$

To answer an equality query, for example, in order to evaluate the equality query " $A=3$ ", the retrieval function for this query is created as $D^{5} \wedge D^{1}$ since the values of $r$ and $s$ is 5 and 1 , respectively. The result of this equality query is the $1^{\text {st }}$ and $6^{\text {th }}$ row. To answer range queries, 2 bitmap vectors identifying each query value can be dynamically created using Eq. (4). Then, bitwise-OR operations are used to obtain the final result. For example, to evaluate the range query " $3 \leq A \leq 13$ ", the retrieval 
http://wjst.wu.ac.th

function for this query is created as $\left(D^{5} \wedge D^{1}\right) \vee\left(D^{5} \wedge D^{0}\right) \vee\left(D^{4} \wedge D^{3}\right) \vee\left(D^{4} \wedge D^{2}\right) \vee\left(D^{4} \wedge D^{1}\right) \vee$ $\left(D^{4} \wedge D^{0}\right) \vee\left(D^{3} \wedge D^{2}\right) \vee\left(D^{3} \wedge D^{1}\right) \vee\left(D^{3} \wedge D^{0}\right) \vee\left(D^{2} \wedge D^{1}\right) \vee\left(D^{2} \wedge D^{0}\right)$

As in the above example, a large number of bitmap vectors and logical operations are performed in order to answer a range query when the range of the query values is wide. Therefore, the performance of traditional range query processing by the dual bitmap index is degraded. In this paper, an algorithm is proposed, called Dual-simRQ, to optimize a range query processing for the dual bitmap index by minimizing the number of bitmap vectors scanned and the number of bitwise operations performed.

\section{Materials and methods}

This section presents the Dual-simRQ algorithm, which is able to simplify the retrieval function of range query processing with the dual bitmap index. This algorithm consists of 4 phases, 1) building the RsJoin table, 2) building the FreqSorting table, 3) simplifying the retrieval function, and 4) generating a new retrieval function, as described in the following.

\begin{tabular}{ccc}
\multicolumn{2}{c}{ RsValue Table } \\
\hline value & $\boldsymbol{r}$ & $\boldsymbol{s}$ \\
\hline 3 & 5 & 1 \\
4 & 5 & 0 \\
5 & 4 & 3 \\
6 & 4 & 2 \\
7 & 4 & 1 \\
8 & 4 & 0 \\
9 & 3 & 2 \\
10 & 3 & 1 \\
11 & 3 & 0 \\
12 & 2 & 1 \\
13 & 2 & 0 \\
\hline
\end{tabular}

(a)

\begin{tabular}{cc}
\multicolumn{2}{c}{ RsJoin Table } \\
\hline unique_r & join_s \\
\hline 5 & $\{1,0\}$ \\
4 & $\{3,2,1,0\}$ \\
3 & $\{2,1,0\}$ \\
2 & $\{1,0\}$ \\
\hline
\end{tabular}

(b)

Figure 2 The result of the temporary RsValue table (a) and the RsJoin table for the range query "3 $\leq A \leq 13 "$ (b).

\section{1) Building the RsJoin table}

Generally, each indexed attribute value is identified by both the values of $r$ and $s$ in the dual bitmap index. When a range query is submitted, an RsValue table is temporarily created by storing a pair of values of $r$ and s corresponding to all the query values. Obviously, each distinct value of $r$ relates to different values of $s$. Therefore, a set of values for $s$ can be created which relate to each distinct value of $r$, where the value of $r$ and associated set of values of $s$ are stored in an RsJoin table. For example, in order to evaluate a range query in the form, " $3 \leq A \leq 13$ ", the RsValue table is firstly created by calculating the values of $r$ and $s$ for the query values between 3 and 13, as shown in Figure 2(a). Subsequently, the RsJoin table is built by finding the distinct values of $r$ and their associated values of $s$, as shown in Figure 2(b). In the first row, the value of $r$ is 5 which relates to the values 1 , and 0 of $s$, which yields set $\{1,0\}$ for $r=5$.

\section{2) Building the FreqSorting table}

The objective of this phase is to find the unique sets of join_s (called unique_s) given by the RsJoin table and their frequency, resulting in a frequency table. Basically, the frequency of unique_s provides an easy combination of the relevant values of $r$ which correspond to unique_s. Furthermore, the number of items in the unique_s has an impact on the reduction of the number of bitwise operations performed 
http://wjst.wu.ac.th

among the relevant bitmap vectors. Therefore, the FreqSorting table is created by arranging the frequency of unique_s from high to low because the values of unique_s with high frequency relate to the number of relevant values of $r$. In addition, the smaller sets of unique_s may possibly be subsets of a larger set. Therefore, in order to eliminate the redundant items, the number of items in each of unique_s is arranged from small to large if the frequency is the same. Figure 3(a) illustrates the frequency belonging to each of unique_s without sorting, which yields sets of $\{1,0\},\{3,2,1,0\}$, and $\{2,1,0\}$ with the frequency of 2,1 , and 1 , respectively. The frequency of set $\{3,2,1,0\}$ and $\{2,1,0\}$ is equal to 1 but the number of items in the latter set is less, i.e., there are 3 items in set $\{2,1,0\}$ while there are 4 items in set $\{3,2,1,0\}$. Therefore, the FreqSorting table consists of the sets of $\{1,0\},\{2,1,0\}$, and $\{3,2,1,0\}$, as shown in Figure 3(b).

\begin{tabular}{cc}
\multicolumn{2}{c}{ Frequency Table } \\
\hline freq & unique_s \\
\hline 2 & $\{1,0\}$ \\
1 & $\{3,2,1,0\}$ \\
1 & $\{2,1,0\}$ \\
\hline
\end{tabular}

(a)

\begin{tabular}{cc}
\multicolumn{2}{c}{ FreqSorting Table } \\
\hline freq & sort_s \\
\hline 2 & $\{1,0\}$ \\
1 & $\{2,1,0\}$ \\
1 & $\{3,2,1,0\}$
\end{tabular}

(b)

Figure 3 The result of the Frequency table (a) and the FreqSorting table for the range query "3 $\leq A \leq 13$ " (b).

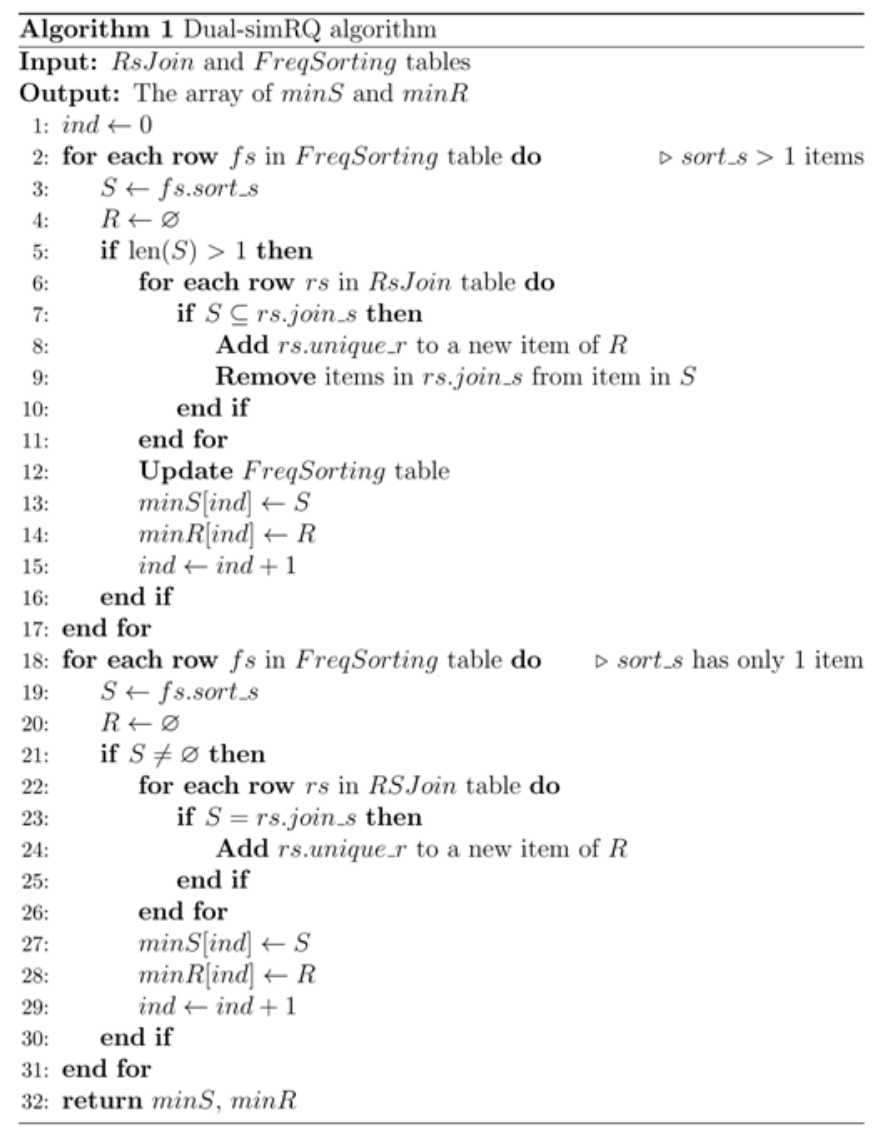

Figure 4 The Dual-simRQ algorithm. 
http://wjst.wu.ac.th

\section{3) Simplifying the retrieval function}

This phase generates a simplification of the retrieval function by utilizing the RsJoin and the FreqSorting tables as an input and produces an array structure of $\min S$ and $\min R$ corresponding to the values of $r$ and $s$, respectively as an output. Figure 4 shows the detail of the Dual-simRQ algorithm. There are 32 steps in the algorithm. In the $1^{\text {st }}$ step, the value of ind is initially set to 0 . In the $2^{\text {nd }}$ to $17^{\text {th }}$ steps, the relevant values of $r$ are combined into a set corresponding to each sort_s if the number of items in the sort_s is more than one. In the $3^{\text {rd }}$ and $4^{\text {th }}$ steps, each sort_s retrieved from the FreqSorting table is assigned to a set $S$ and an empty set is assigned to a set $R$ in order to hold the sort_s, and its relevant values of $r$, respectively. In the $5^{\text {th }}$ to $11^{\text {th }}$ steps, the values of unique_r in the RsJoin table are gathered into a set if the set $S$ is a subset of each join_s in the RsJoin table, which yields a set $R$. Then, the join_s is updated by removing the redundant items from $S$ at the $9^{\text {th }}$ step. In the $12^{\text {th }}$ step, the FreqSorting table is updated by removing the redundant items from $S$ if the set $S$ is a subset of each sort_s. In the $13^{\text {th }}$ and $14^{\text {th }}$ steps, the results of sets $S$ and $R$ are assigned to $\min S$ and $\min R$, respectively, at the index of ind. The value of ind is increased by 1 at the $15^{\text {th }}$ step. In the $18^{\text {th }}$ to $31^{\text {st }}$ steps, the sets sort $s$ containing only one item are executed by repeating the same process to find the relevant values of $r$ in the RsJoin table corresponding to the each of set sort_s. Consequently, each index of the array $\min S$ and $\min R$ consists of sets of $S$ and sets of $R$, respectively, as shown in Figure 5.

\begin{tabular}{ccc}
\multicolumn{3}{c}{ MinTerm } \\
\hline ind & minS $[\boldsymbol{i n d}]$ & $\boldsymbol{m i n R}[\boldsymbol{i n d}]$ \\
\hline 0 & $\{1,0\}$ & $\{5,4,3,2\}$ \\
1 & $\{3,2\}$ & $\{4\}$ \\
2 & $\{2\}$ & $\{3\}$ \\
\hline
\end{tabular}

Figure 5 The result of array $\min S$ and $\min R$ for the range query " $3 \leq A \leq 13 "$.

\section{4) Generating a new retrieval function phase}

In this phase, a new retrieval function is generated by retrieving all the items within the array $\min S$ and $\min R$ at every index, as shown in Figure 5. Each item of $\min S$ refers to values of $s$ while each item of $\min R$ refers to values of $r$. These items within $\min S$ and $\min R$ relate to an identifier of a bitmap vector for the dual bitmap index. Therefore, all the related bitmap vectors specified by items in $\min S$ are performed by a bitwise-OR operation as well as all items in $\min R$. Then, each result of $\min S$ and $\min R$ is performed in a bitwise-AND operation to obtain a sub-result of at any index value. For example, at the ind $=0$, $\operatorname{minS}[0]$ consists of 2 values (i.e., 1,0 ) while $\min R[0]$ consists of 4 values (i.e., 5,4 , 3, and 2). The bitwise-OR operations are performed between bitmap vectors $D^{0}$ and $D^{1}$, to give the result for minS[0]. Subsequently, the bitwise-OR operations are performed among bitmap vectors $D^{5}, D^{5}, D^{4}$, and $D^{2}$, to give the result for $\min R[0]$. After that, the results of $\min S[0]$ and $\min R[0]$ are performed in a bitwise-AND to obtain the sub-result for ind $=0$. Finally, the end result is obtained by performing bitwise-OR operations among all the sub-results. Therefore, the retrieval function for the range query " $3 \leq A \leq 13$ " is $\left(\left(D^{1} \vee D^{0}\right) \wedge\left(D^{5} \vee D^{4} \vee D^{3} \vee D^{2}\right)\right) \vee\left(\left(D^{3} \vee D^{2}\right) \wedge D^{4}\right) \vee\left(D^{2} \wedge D^{3}\right)$.

\section{Results and discussion}

This section presents a theoretical analysis and experimental results in which the range query processing time for 2 algorithms, the traditional range query processing (Dual-tradRQ) and the DualsimRQ, was compared. 
http://wjst.wu.ac.th

Table 1 A comparative study of Dual-tradRQ and Dual-simRQ for range query processing

\begin{tabular}{cccc}
\hline \multirow{2}{*}{$\begin{array}{c}\text { Bitmap index } \\
\text { algorithm }\end{array}$} & Space requirement & \multicolumn{2}{c}{ Range query processing time } \\
\cline { 3 - 4 } & $\begin{array}{c}\text { Number of bitmap vectors } \\
\text { scanned }\end{array}$ & $\begin{array}{c}\text { Number of Boolean } \\
\text { operation used }\end{array}$ \\
\hline Dual-tradRQ & {$[\sqrt{2 C+0.25}+0.5]$} & $2 k$ & $2 k-1$ \\
Dual-simRQ & {$[3,2 k]$} & {$[2,2 k-1]$} \\
\hline
\end{tabular}

$C$ is the cardinality of the attribute and $k$ is the range of query values

Table 1 shows the theoretical analysis of the 2 algorithms for range query processing based on a dual bitmap index. Both the algorithms require the same number of bitmap vectors, i.e., $[\sqrt{2 C+0.25}+$ 0.5 . For the performance of range query processing, the Dual-tradRQ scans $2 k$ bitmap vectors and uses $2 k-1$ Boolean operators. The number of bitmap vectors scanned for the Dual-simRQ varies from 3 to $2 k$ bitmap vectors and the number of Boolean operations used also varies between 2 and $2 k-1$. For example, for Dual-simRQ, if it is assumed that there are 2 query values submitted and the former retrieval function can be reduced, this implies that those query values have the same value of $r$. Therefore, the minimum number of bitmap vectors scanned is 3 and the minimum number of Boolean operations used is 2 (1 AND and $1 \mathrm{OR})$. On the other hand, if the retrieval function cannot be reduced, this means that the query values have different values of $r$, in which case, the number of bitmap vectors scanned is 4 and the number of Boolean operations used is 3 (i.e., 2 ANDs and 1 OR).

The experiments were conducted on a $3.20 \mathrm{GHz}$ Intel ${ }^{\circledR} \mathrm{Core}^{\mathrm{TM}}$ i5-4570HQ with a $4.00 \mathrm{~GB}$ main memory, running on 64-bit Windows 10 as an operating system. The range query processing time includes the CPU and I/O time required to answer range queries. In particular, the CPU time for the DualsimRQ includes the processes involved in simplifying the retrieval function which occupies a small part of execution time.

The experiment used the TPC-H benchmark data set retrieved from [27]. This benchmark consists of 8 tables. The experiment was conducted on the tables PART and ORDERS which contain 20,000,000 and 150,000,000 records, respectively. Three representative attributes were used from table PART and one representative attribute was used from table ORDERS, as follows (the values in parentheses indicate the cardinality of the corresponding attribute). The attributes selected were: BRAND (25), SIZE (50), and TYPE (150) from the table PART, and attribute CLERK $(1,000)$ from the table ORDERS. This provided different cardinalities ranging from low to high. In addition, 5 different range value ratios ranging from 10 to $80 \%$ of the cardinality, representing the number of continuous query values in a range query, were selected.

Figure 6 highlights the abovementioned theoretical analysis by comparing the Dual-simRQ algorithm with the Dual-tradRQ for evaluating range queries on the selected attributes with respect to five range ratios with 4 different cardinalities. The Dual-tradRQ does not perform any simplification of the retrieval function. Figures 6(a) - 6(d) show the range query processing time for cardinalities of 25, 50, 150 , and 1,000, respectively. When the range ratio increased, the overall query processing time of DualtradRQ was significantly increased while that of Dual-simRQ increased only slightly. When the cardinality and the range ratio are low and the retrieval function can be slightly minimized, then, the CPU time used by Dual-simRQ is slightly more than that used by Dual-tradRQ. However, when both the cardinality and range ratio are high, the CPU time used by Dual-simRQ is less than that of Dual-tradRQ. Furthermore, the Dual-simRQ uses less I/O time than Dual-tradRQ for all cardinality and range ratios, which implies that the number of bitmap vectors scanned by Dual-simRQ is less than the number of bitmap vectors scanned by Dual-tradRQ. Overall, the experimental results show that the proposed algorithm, Dual-simRQ, is much faster than Dual-tradRQ in every range ratio for all selected attributes, indicating that the Dual-simRQ is able to optimize the range query processing of the dual bitmap index for every range ratios and different ranges of cardinality. 


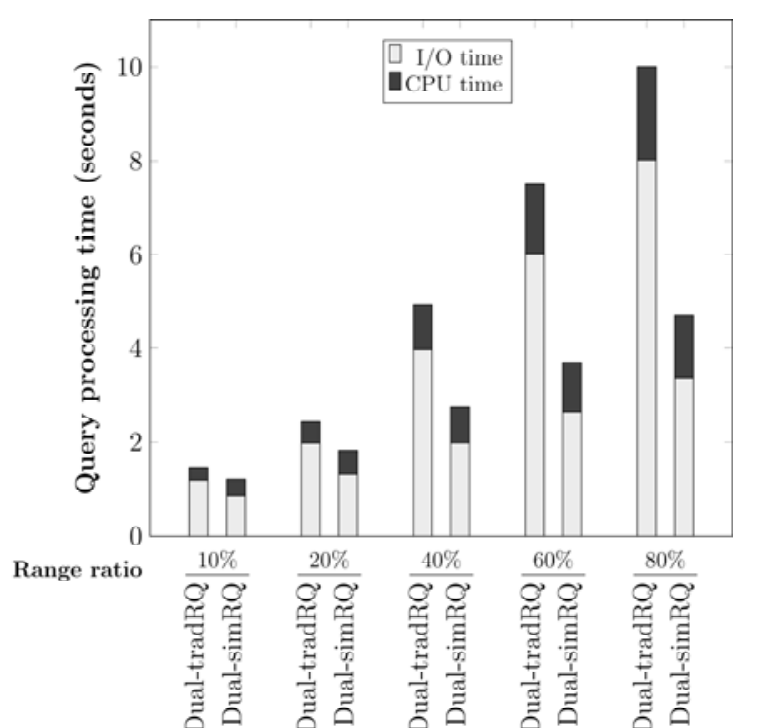

(a)

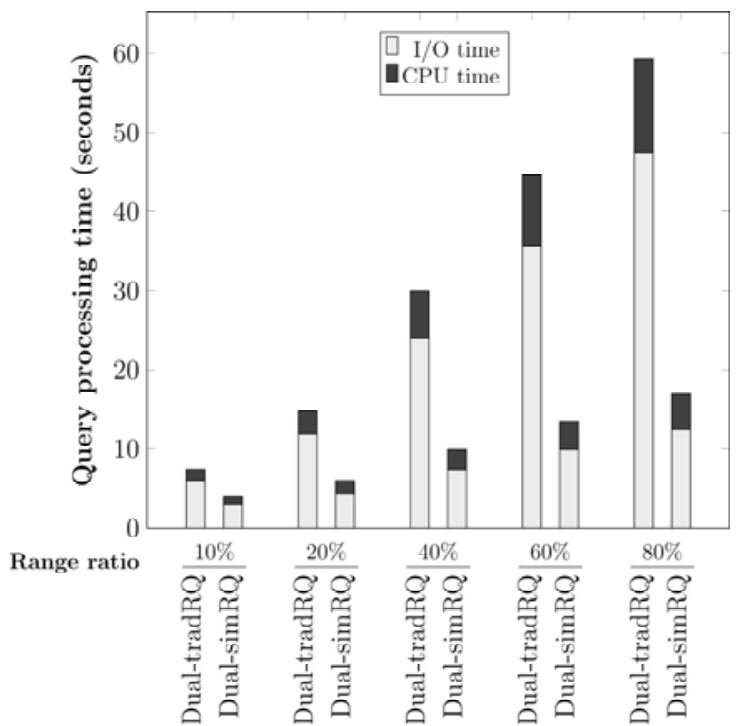

(c)

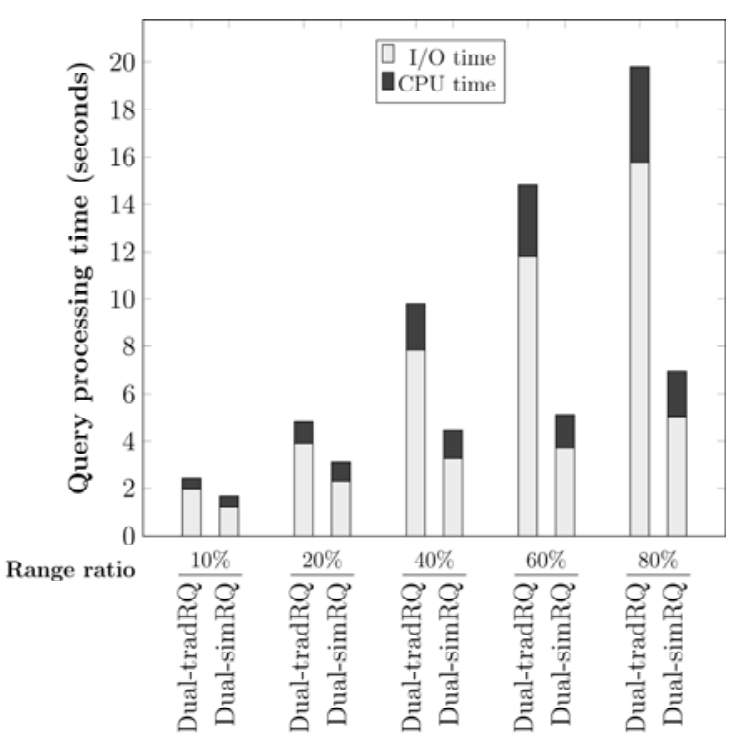

(b)

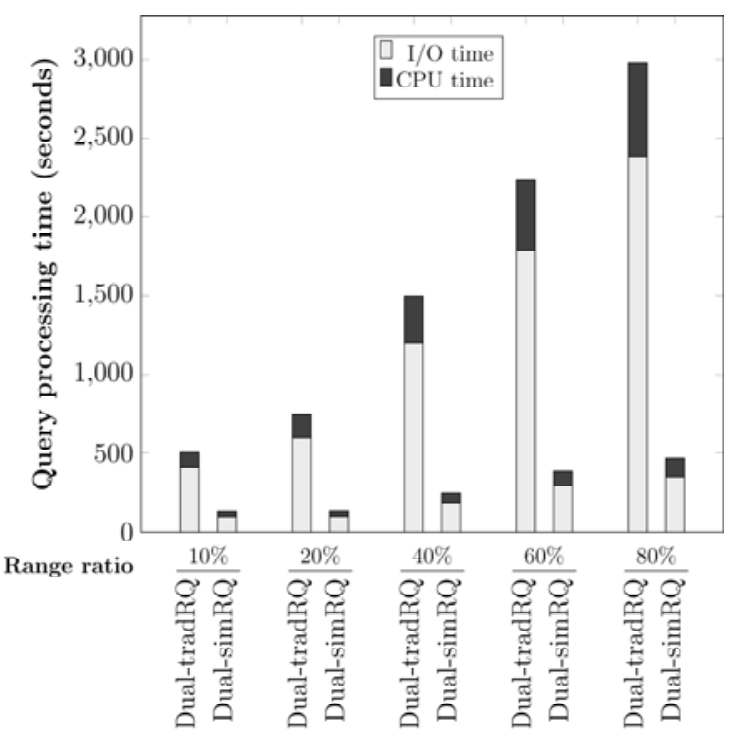

(d)

Figure 6 The comparison of range query processing time with 5 range value ratios on the attribute BRAND with $C=25$ (a), attribute SIZE with $C=50$ (b), attribute TYPE with $C=150$ (c), and attribute CLERK with $C=1,000$ (d).

\section{Conclusions}

With the availability of significantly increasing amounts of data emerges both the challenge and the opportunity to discover large amounts of valuable knowledge. However, the requirement for data storage and the time required to process queries on such large amounts of data has become a major problem when answering complex queries. Various bitmap-based indexes exist which aim to reduce the storage requirement without sacrificing query processing time by performing fast Boolean operations at the index 
http://wjst.wu.ac.th

level. The dual bitmap index optimizes the space vs. time trade-off when answering equality queries. However, the dual bitmap index takes a long time when answering range queries. In this paper, the DualsimRQ algorithm was introduced, which is capable of reducing the query processing time for range queries based on a dual bitmap index. The Dual-simRQ algorithm eliminates the unnecessary scanning of bitmap vectors and performing of Boolean operations. The results of the experiment described show that the Dual-simRQ is much faster than the Dual-tradRQ algorithm. In addition, future research will investigate a new encoding bitmap index algorithm to optimize the performance of all kinds of selective queries in terms of the space vs. time trade-off.

\section{Acknowledgements}

This research was financially supported by Faculty of Science Research Fund: 158002, Prince of Songkla University, Hat Yai, Songkhla.

\section{References}

[1] S Sagiroglu and D Sinanc. Big data: A review. In: Proceedings of 2013 International Conference on Collaboration Technologies and Systems, San Diego, USA, 2013, p. 42-7.

[2] S Chaudhuri. What next? A half-dozen data management research goals for big data and the cloud. In: Proceedings of the $31^{\text {st }}$ ACM SIGMOD-SIGACT-SIGAI Symposium on Principles of Database Systems, Scottsdale, Arizona, USA, 2012, p. 1-4.

[3] A Katal, M Wazid and R H Goudar. Big data: Issues, challenges, tools and good practices. In: Proceedings of $20136^{\text {th }}$ International Conference on Contemporary Computing, Noida, India, 2013, p. 404-9.

[4] S Chaudhuri and U Dayal. An overview of data warehousing and OLAP technology. ACM SIGMOD Rec. 1997; 26, 65-74.

[5] CY Chan and YE Ioannidis. Bitmap index design and evaluation. ACM SIGMOD Rec. 1998; 27, 355-66.

[6] K Wu, A Shoshani and K Stockinger. Analyses of multi-level and multi-component compressed bitmap indexes. ACM Trans. Database Syst. 2010; 35, 1-52.

[7] K Kambatla, G Kollias, V Kumar and A Grama. Trends in big data analytics. J. Parallel Distr. Comput. 2014; 74, 2561-73.

[8] X Wu, X Zhu, GQ Wu and W Ding. Data mining with big data. IEEE Trans. Knowl. Data Eng. 2014; 26, 97-107.

[9] K Stockinger and K Wu. Bitmap indices for data warehouses. Data Warehouses OLAP Concepts Architect. Solut. 2007; 1, 157-78.

[10] ZQ Abdulhadi, Z Zuping and HI Housien. Bitmap index as effective indexing for low cardinality column in data warehouse. Int. J. Comput. Appl. 2013; 68, 38-42.

[11] LJ Gosink, K Wu, EW Bethel, JD Owens and KI Joy. 2008, Bin- Hash Indexing: A Parallel Method for Fast Query Processing, Technical Report. Laurence Berkeley National Laboratories, USA.

[12] Y Mei, K Ji and F Wang. A survey on bitmap index technologies for large-scale data retrieval. In: Proceedings of $20136^{\text {th }}$ International Conference on Intelligent Networks and Intelligent Systems, Shenyang, China, 2013, p. 316-9.

[13] Z Chen, Y Wen, J Cao, W Zheng, J Chang, Y Wu, G Ma, M Hakmaoui and G Peng. A survey of bitmap index compression algorithms for big data. Tsinghua Sci. Tech. 2015; 20, 100-15.

[14] PO Neil and D Quass. Improved query performance with variant indexes. ACM SIGMOD Rec. 1997; 26, 38-49.

[15] CY Chan and YE Ioannidis. An efficient bitmap encoding scheme for selection queries. ACM SIGMOD Rec. 1999; 28, 215-26.

[16] M Stabno and R Wrembel. RLH: Bitmap compression technique based on run-length and huffman encoding. Inform. Syst. 2009; 34, 400-14. 
http://wjst.wu.ac.th

[17] S Kim, J Lee, SR Satti and B Moon. SBH: Super byte-aligned hybrid bitmap compression. Inform. Syst. 2016; 62, 155-68.

[18] KL Wu and S Yu. Range-based bitmap indexing for high cardinality attributes with skew. In: Proceedings of the $22^{\text {nd }}$ Annual International Computer Software and Applications Conference, Vienna, Austria, 1998, p. 61-6.

[19] MC Wu and AP Buchmann. Encoded bitmap indexing for data warehouses. In: Proceedings of $14^{\text {th }}$ International Conference on Data Engineering, Orlando, USA, 1998, p. 220-30.

[20] S Vanichayobon, J Manfuekphan and L Gruenwald. Scatter bitmap: Space-time efficient bitmap indexing for equality and membership queries. In: Proceedings of 2006 IEEE Conference on Cybernetics and Intelligent Systems, Bangkok, Thailand, 2006, p. 1-6.

[21] W Weahama, S Vanichayobon and J Manfuekphan. Using data clustering to optimize scatter bitmap index for membership queries. In: Proceedings of 2009 International Conference on Computer and Automation Engineering, Bangkok, Thailand, 2009, p. 174-8.

[22] N Wattanakitrungroj and S Vanichayobon. Dual bitmap index: Space-time efficient bitmap index for equality and membership queries. In: Proceedings of 2006 International Symposium on Communications and Information Technologies, Bangkok, Thailand, 2006, p. 568-73.

[23] GR Alam, MY Arafat, M Kamal and U Iftekhar. A new approach of dynamic encoded bitmap indexing technique based on query history. In: Proceedings of $5^{\text {th }}$ International Conference on Electrical and Computer Engineering, Dhaka, Bangladesh, 2008, p. 974-9.

[24] J Sainui, S Vanichayobon and N Wattanakitrungroj. Optimizing encoded bitmap index using frequent itemsets mining. In: Proceedings of 2008 International Conference on Computer and Electrical Engineering, Phuket, Thailand, 2008, p. 511-5.

[25] A Keawpibal, N Wattanakitrungroj and S Vanichayobon. Enhanced encoded bitmap index for equality query. In: Proceedings of the $8^{\text {th }}$ International Conference on Computing Technology and Information Management, Seoul, South Korea, 2012, p. 293-8.

[26] N Keawpibal, J Duangsuwan, W Wettayaprasit, L Preechaveerakul and S Vanichayobon. DistEQ: Distributed equality query processing on encoded bitmap index. In: Proceedings of the $12^{\text {th }}$ International Joint Conference on Computer Science and Software Engineering, Songkhla, Thailand, 2015, p. 309-14.

[27] TPC-H: A Decision Support Benchmark. Available at: http://www.tpc.org/tpch, accessed November 2017. 